\title{
EVIDÊNCIAS CIENTÍFICAS ACERCA DA PARALISIA CEREBRAL INFANTIL
}

\author{
Virna Ribeiro Feitosa Cestari1, Islene Victor Barbosa², Zuíla Maria de Figueiredo Carvalho ${ }^{3}$, \\ Elizabeth Mesquita $\mathrm{Melo}^{4}$, Rita Mônica Borges Studart ${ }^{5}$
}

\begin{abstract}
RESUMO: Revisão integrativa da literatura que objetivou descrever as evidências científicas disponíveis acerca da paralisia cerebral infantil. A busca dos artigos se deu nas bases SciELO, LILACS e MEDLINE, nos meses de maio a setembro de 2012. A amostra foi constituída por 25 estudos publicados, nos idiomas português e/ou inglês, entre janeiro de 2006 e abril de 2012. A análise das publicações identificou oito eixos temáticos sobre o tema: considerações gerais acerca da patologia, classificação do grau de comprometimento motor e cognitivo, conhecimento dos pais acerca da paralisia cerebral, o impacto da doença para as mães/ familiares e crianças, avaliação e promoção da qualidade de vida das mães/crianças, assistência à criança com paralisia cerebral, e inclusão social da criança com paralisia cerebral. Constatou-se reduzido número de artigos publicados relacionados à assistência de enfermagem à criança com paralisia cerebral, evidenciando a necessidade de mais estudos sobre o tema no Brasil e no mundo. DESCRITORES: Enfermagem; Paralisia cerebral; Criança; Cuidados de enfermagem.
\end{abstract}

\section{SCIENTIFIC EVIDENCE REGARDING INFANT CEREBRAL PALSY}

ABSTRACT: This integrative literature review aimed to describe the scientific evidence available regarding infant cerebral palsy. The search for articles took place in the SciELO, LILACS and MEDLINE databases, in May-September 2012. The sample was made up of 25 published studies, in the Portuguese and/or English languages, between January 2006 and April 2012. The analysis of the publications identified eight thematic axes regarding the issue: general considerations about the pathology, classification of the extent of motor and cognitive compromise, the parents' knowledge regarding the cerebral palsy, the condition's impact for the mothers/family members and children, evaluation and promotion of quality of life of the mothers/children, care for the child with cerebral palsy, and social inclusion of the child with cerebral palsy. It was observed that there is a small number of articles published related to nursing care for the child with cerebral palsy, evidencing the need for further studies on the issue in Brazil and worldwide. DESCRIPTORS: Nursing; Cerebral palsy; Child; Nursing care.

\section{EVIDENCIAS CIENTÍFICAS ACERCA DE LA PARÁLISIS CEREBRAL INFANTIL}

RESUMEN: Revisión integrativa de literatura que tuvo la finalidad de describir las evidencias científicas disponibles acerca de la parálisis cerebral infantil. La búsqueda de los artículos ocurrió en las bases SCIELO, LILACS y MEDLINE, en los meses de mayo a septiembre de 2012. La muestra fue constituida por 25 estudios publicados, en los idiomas portugués y/o inglés, entre enero de 2006 y abril de 2012. El análisis de las publicaciones identificó ocho ejes temáticos: consideraciones generales acerca de la patología, clasificación del grado de compromiso motor y cognitivo, conocimiento de los padres acerca de la parálisis cerebral, el impacto de la enfermedad para las madres/familiares y niños, evaluación y promoción de la cualidad de vida de las madres/niños, asistencia al niño con parálisis cerebral, e inclusión social del niño con parálisis cerebral. Se constató que hay reducido número de artículos publicados asociados a la asistencia de enfermería al niño con parálisis cerebral, evidenciando la necesidad de más estudios sobre el tema en Brasil y en mundo.

DESCRIPTORES: Enfermería; Parálisis cerebral; Niño; Cuidados de enfermería.

\footnotetext{
${ }^{1}$ Acadêmica do Curso de Graduação em Enfermagem da Universidade de Fortaleza - UNIFOR. Membro do Núcleo de Pesquisa e Extensão em Enfermagem Neurológica da Universidade Federal do Ceará - NUPEN UFC.

${ }^{2}$ Enfermeira. Doutora em Enfermagem. Professora do Curso de Graduação em Enfermagem da UNIFOR. Membro do NUPEN UFC. ${ }^{3}$ Enfermeira. Pós-Doutora em Enfermagem. Professora do Programa de Pós-Graduação do Departamento de Enfermagem da Faculdade de Farmácia, Odontologia e Enfermagem da UFC. Coordenadora do NUPEN UFC.

${ }^{4}$ Enfermeira. Doutora em Enfermagem. Professora do Curso de Graduação em Enfermagem da UNIFOR.

${ }^{5}$ Enfermeira. Doutora em Enfermagem. Professora do Curso de Enfermagem da UNIFOR. Membro do NUPEN UFC.
}

Autor correspondente:

Virna Ribeiro Feitosa Cestari

Universidade de Fortaleza

Rua Núbia Barrocas, 1280 - 60821-770 - Fortaleza-CE-Brasil

E-mail: virna.ribeiro@hotmail.com
Recebido: 23/04/2013

Aprovado: 01/11/2013 


\section{INTRODUÇÃO}

A Encefalopatia Crônica Não Progressiva da Infância ou Paralisia Cerebral (PC) é uma doença do sistema nervoso central que interfere no desenvolvimento motor normal da criança, caracterizada por um transtorno do tônus postural e do movimento ${ }^{(1)}$. Consiste em um distúrbio não progressivo resultante de uma lesão que ocorre no cérebro quando este é imaturo, ainda em desenvolvimento fetal ou infantil ${ }^{(2)}$.

A incidência de casos de $\mathrm{PC}$ na populaçãoé de dois em cada mil nascidos vivos, em países em desenvolvimento chega a sete por mil nascidos vivos. No Brasil, é estimada a ocorrência de 30.000 a 40.000 casos novos por ano ${ }^{(3)}$.

Os déficits motores da PC incluem fenômenos negativos e fenômenos positivos. Os negativos envolvem fraqueza, fadiga e incoordenação; são fenômenos positivos a espasticidade, clono, rigidez e espasmos, os quais que podem levar à rigidez muscular, imparidade funcional e atrofia; quando não tratada pode progredir para fibrose muscular, contraturas e deformidades musculoesqueléticas ${ }^{(4)}$. Existe, ainda, elevado índice de alterações na sensação, percepção, cognição, comunicação e comportamento, além de crises convulsivas e problemas musculoesqueléticos secundários ${ }^{(5)}$.

O manejo de crianças com PC envolve a organização de equipes multiprofissionais que buscam minimizar o impacto desta patologia no seu desenvolvimento global. Por conseguinte, faz-se necessário oferecer possibilidade de intervenções que considerem as diferenças individuais e permita o aprimoramento de suas potencialidades ${ }^{(6)}$.

A Enfermagem é uma profissão que tem no cuidado a especificidade e a essência do seu trabalho, é responsável pelo acolhimento, conforto e bem estar dos pacientes e de suas famílias. Nesta perspectiva, o enfermeiro pode levá-los a desenvolver habilidades e contribuir para sua inclusão social( ${ }^{(7)}$.

Este estudo torna-se relevante para a enfermagem, pois pretende trazer subsídios para a divulgação das evidências da prática clínica abordadas nas pesquisas com a temática, bem como proporcionar a divulgação das evidências científicas disponíveis para a avaliação de opções e tomada de decisão na assistência ao paciente. A PC é assunto escasso nas grades curriculares e, frente sua alta prevalência, despertou-se o interesse para conhecer os aspectos abordados sobre o tema, agravo que requer exame, tratamento e acompanhamento especializado. Nessa perspectiva, tem-se como objetivo descrever as evidências científicas disponíveis acerca da paralisia cerebral infantil.

\section{MÉTODO}

Este estudo caracteriza-se como uma revisão integrativa da literatura, método que tem a finalidade de reunir e sintetizar resultados de pesquisas sobre um delimitado tema ou questão, de maneira sistemática e ordenada, contribuindo para o aprofundamento do conhecimento do tema investigado ${ }^{(8)}$.

$\mathrm{Na}$ construção desta revisão percorreram-se seis etapas: elaboração da questão norteadora; estabelecimento de critérios de inclusão e exclusão; busca na literatura; definição das informações a ser extraída dos estudos selecionados; avaliação dos estudos incluídos na revisão integrativa; e interpretação dos resultados $^{(8)}$. A questão norteadora da pesquisa foi: quais as evidências científicas sobre a paralisia cerebral infantil? Foram consultadas as bases de dados Literatura Latino-Americana e do Caribe em Ciências da Saúde (LILACS), Scientific Eletronic Library Online (SciELO) e Medical Literature Analysis and Retrieval System Online (MEDLINE), o acesso foi por meio da Biblioteca Virtual da Saúde (BVS).

A busca ocorreu no período de maio a setembro de 2012, utilizando-se os Descritores em Ciências da Saúde: enfermagem, paralisia cerebral, criança e cuidados de enfermagem; e os do Medical Subject Headings: nursing, cerebral palsy, child e nursing care. Os descritores selecionados foram combinados entre si, acrescentando-se o termo 'and' entre eles, para que os resultados da busca atendessem aos objetivos do estudo.

Os critérios de inclusão das publicações foram: indexadas nas bases de dados selecionadas; publicadas no período de janeiro de 2006 a abril de 2012; nos idiomas português e/ou inglês e disponíveis eletronicamente. Foram excluídos do estudo aqueles publicados em eventos científicos. Para a coleta de dados dos artigos foi desenvolvido um formulário pelas pesquisadoras, o qual foi preenchido com dados de cada artigo selecionado, tais como título, autoria, país de origem, nome do periódico, objetivos e resultados. Os dados alimentaram planilha do programa Excel e procedeu-se a análise descritiva dos conteúdos.

\section{RESULTADOS}

Foram identificados 705 estudos, dos quais 120 foram pré-selecionados por meio da leitura dos respectivos títulos e resumos, sendo 75 da SciELO, 25 da LILACS e 20 da MEDLINE. Dos 120 estudos pré-selecionados, 95 foram excluídos pelos seguintes 
motivos: 18 estavam duplicados na base de dados e entre as bases de dados, 32 não tinham relação com o tema, cinco não correspondiam aos idiomas dos critérios de seleção e 15 não estavam disponíveis na íntegra. Dessa forma, totalizou-se uma amostra final de 25 estudos nessa revisão integrativa.

Verificou-se um número crescente de publicações a partir de 2009, representado por oito artigos (32\%). Em relação ao idioma de publicação, o português predominou com 16 artigos (64\%), conforme exposto nas Tabelas 1 e 2 .

Tabela 1 - Identificação dos estudos publicados sobre paralisia cerebral na infância na literatura brasileira entre jan. 2006 - abr. 2012. Fortaleza, 2012

\begin{tabular}{lcc}
\hline Primeiro autor & $\begin{array}{c}\text { Ano de } \\
\text { publicação* }\end{array}$ & Profissão \\
\hline Gondim KM & 2012 & Enfermagem \\
Souza KES & 2011 & Fisioterapia \\
Ribeiro MFM & 2011 & Enfermagem \\
Oliveira ANA & 2010 & Fisioterapia \\
Prudente COL & 2010 & Enfermagem \\
Dantas MAS & 2010 & Enfermagem \\
Vasconcelos VM & 2010 & Enfermagem \\
Pfeifer LI & 2009 & Medicina \\
Brasileiro IC & 2009 & Enfermagem \\
Silveira RD & 2009 & Enfermagem \\
Massi G & 2009 & Fonoaudiologia \\
Milbrath VM & 2009 & Enfermagem \\
Inácio IF & 2008 & Enfermagem \\
Chagas PDC & 2008 & Fisioterapia \\
Lotitto FZ & 2008 & Enfermagem \\
Teixeira-Arroyo C & 2007 & EducaçãoFísica \\
\hline
\end{tabular}

* Bases de dados LILACS, SciELO, MEDLINE
Observou-se que a Enfermagem foi a profissão que mais publicou artigos relacionados à temática na literatura nacional; em se tratando da internacional, verificou-se uma igualdade entre as publicações da Enfermagem e da Medicina.

Quanto ao delineamento, identificou-se pesquisas do tipo descritiva e descritiva-exploratória, revisão bibliográfica e revisão sistemática, transversal, estudo de caso, experimental e observacional. Quanto à abordagem metodológica, evidenciou-se o predomínio da abordagem qualitativa, 10(40\%), seguida da quantitativa com $8(32 \%)$; e duas( $8 \%$ ) quanti-qualitativa. Verificou-se que cinco estudos (20\%) não citaram a abordagem utilizada e não foi possível verificar com apresentação dos resultados.

Dentro da classificação hierárquica metodológica proposta pela Prática Baseada em Evidências ${ }^{(9)}$, quatro $\operatorname{artigos}(16 \%)$ foram classificados no nível 3; houve equivalência entre os níveis 4 e 5 , com 10 artigos cada (40\%); e um artigo foi classificado no nível 6 (4\%).

A partir dos artigos estudados, foram identificadas 8 eixos temáticos prevalentes acerca PC infantil: considerações gerais acerca da patologia (20\%), classificação do grau de comprometimento motor e cognitivo (20\%), conhecimento dos pais acerca da PC (4\%), o impacto da doença para as mães/familiares e crianças (20\%), Avaliação e promoção da qualidade de vida das mães/ crianças (24\%), Assistência à criança com PC (8\%) e Inclusão social da criança com PC (4\%).

\section{DISCUSSÃO}

Observou-se que a produção científica nacional sobre PC é quantitativamente maior quando comparada com a internacional, neste estudo. Das temáticas identificadas, verifica-se a predominância daquelas

Tabela 2 - Identificação dos estudos publicados sobre paralisia cerebral na infância na literatura internacional entre jan. 2006 - abr. 2012. Fortaleza, 2012

\begin{tabular}{lccc}
\hline Primeiro autor & Ano de publicação* & Profissão & País de origem \\
\hline Reid SM & 2011 & Medicina & Austrália \\
Sigurdardottir S & 2011 & Medicina & Noruega \\
Parkes J & 2010 & Enfermagem & Irlanda do Norte \\
Parkes J & 2009 & Enfermagem & Irlanda do Norte \\
Buran CF & 2009 & Enfermagem & Estados Unidos \\
Haak P & 2009 & Medicina & Estados Unidos \\
Himpens E & 2008 & Fisioterapia & Bélgica \\
Singh BK & 2006 & Enfermagem & Inglaterra \\
Jan MMS & 2006 & Medicina & Arábia Saudita \\
\hline
\end{tabular}

* Bases de dados LILACS, SciELO, MEDLINE 
relacionadas ao impacto da doença para as mães/familiares e crianças, avaliação e promoção da qualidade de vida das mães/crianças, considerações gerais acerca da patologia e classificação do grau de comprometimento motor e cognitivo. Poucos são os estudos que evidenciam os cuidados de enfermagem direcionados à criança com PC, necessitando-se, portanto, de mais estudos.

A exploração dessa temática é de extrema importância para a profissão, pois o cuidado de enfermagem é essencial e envolve muitas responsabilidades atreladas à complexidade da patologia ${ }^{(10)}$. A PC encontra-se entre as desordens neurológicas mais comuns do neurodesenvolvimento, seguida do autismo e do retardo mental ${ }^{(11)}$.

Muitos autores afirmam que não é possível determinar a incidência da PC devido a sua heterogenia etiológica, diagnóstico precoce difícil e pelo fato de não ser uma doença de notificação compulsória ${ }^{(12-14)}$. Contudo, pesquisadores ${ }^{(2)}$ afirmam que a incidência no Brasil é alta, evidenciando a necessidade de um maior conhecimento acerca da doença; apesar da melhoria dos recursos médicos, a prevalência mundial da PC não diminuiu de forma satisfatória ${ }^{(15)}$.

Através dos artigos analisados percebe-se os danos e prejuízos que a $P C$ causa às crianças e aos familiares ${ }^{(5,16-18)}$; a disfunção motora resulta na incapacidade e limitação do indivíduo em desempenhar atividades e tarefas do seu cotidiano e de sua família. O impacto da disfunção é mais significativo nas atividades que exigem mudança e manutenção da posição do corpo no espaço ${ }^{(4,19-20)}$.

Em relação à mobilidade funcional, foi criado o Sistema de Classificação da Função Motora, que classifica a situação da criança diagnosticada com PC por níveis, variando de I -crianças com mínimo ou nenhuma disfunção relativa à mobilidade, à $\mathrm{V}$ - crianças que são totalmente dependentes e necessitam de ajuda para se movimentar. Esta classificação direciona para uma escolha de avaliações e no planejamento das intervenções ${ }^{(21)}$.

Quando relacionada ao tipo motor, autores destacam predominância do nível I em crianças hemiplégicas, nível III em diplégicas e nível V em quadriplégicas $^{(22)}$. De acordo com pesquisadores ${ }^{(23)}$, a classificação da gravidade do comprometimento motor e o reconhecimento da presença de qualquer doença, ou deficiência associada, são importantes descritores adicionais.

A capacidade do uso da linguagem depende do nível da habilidade motora, cognitiva e sensorial. A maioria das crianças com PC é capaz de expressar sentenças e muitas possuem cognição verbal normal. Aquelas com dificuldade verbal frequentemente possuem deficiências múltiplas ${ }^{(17)}$. Várias são as manifestações e complicações associadas a PC, como retardo mental, epilepsia, problemas com alimentação, nutrição e crescimento, disfunção intestinal, distúrbios do sono, sialorreia, perda auditiva, anormalidades visuais e ortopédicas ${ }^{(11)}$. As crianças podem apresentar, ainda, disfunção urinária caracterizada pela perda do controle da bexiga ${ }^{(24)}$.

Entre os temas encontrados nas publicações da enfermagem, verificou-se a predominância daqueles relacionados ao impacto da doença e a prestação da assistência à criança e familiares; avaliação o conhecimento dos pais acerca da doença; e a promoção da inclusão social da criança com PC. A exploração desses temas é de extrema importância para a profissão, visto ser o enfermeiro promotor da saúde e ter como base para suas ações o cuidado humanizado e holístico.

A assistência de enfermagem para crianças com PC devem transmitir aceitação, afeição, amizade e promover na criança um sentimento de confiança ${ }^{(3)}$. $\mathrm{O}$ enfermeiro tem participação ativa na reabilitação e nos elementos decisivos nos processos interacionais cliente/família/equipe, a fim de que os recursos utilizados na promoção da saúde, prevenção de complicações, orientação para o auto-cuidado, voltados para o resgate da autoestima e autonomia funcional, sejam determinantes para viabilizar o desempenho das atividades da vida diária ${ }^{(25)}$.

Autores evidenciam o impacto do diagnóstico de uma criança portadora de PC para a família ${ }^{(1,26)}$; quando o diagnóstico e prognóstico não são revelados com cautela, podem levar a postergação do início do tratamento, prejudicando a criança. $O$ pouco conhecimento dos pais a respeito do diagnóstico, e suas repercussões na vida da criança com PC, é um fator limitante da participação deles no tratamento, na educação e no cuidado dessas crianças ${ }^{(6)}$.

Estudos demonstraram que os profissionais da saúde, durante a apresentação do diagnóstico às mães, fizeram uso de termos técnicos, dificultando o entendimento. Os autores também afirmaram que a comunicação do diagnóstico não trouxe os esclarecimentos indispensáveis para conscientizar os familiares a respeito da doença ${ }^{(1,26)}$

Uma atenção especial deve ser dada às mães de crianças portadoras de necessidades especiais, visto que elas são mais propensas a sofrer de estresse, ansiedade e depressão, o que dificulta o cuidado à criança, repercutindo de maneira negativa no seu processo de desenvolvimento e crescimento $^{(27)}$. 
Estudo evidenciou que a percepção das mães acerca da qualidade de vida de seus filhos com PC está associada a aspectos financeiros, bem como ao desenvolvimento de atividades da vida diária. Segundo os autores, as mães referenciaram uma qualidade de vida ruim do filho e colocaram-se como responsáveis; elas relataram, ainda, que seus filhos são afetados pelo preconceito e processo de negação da criança com deficiência, interferindo na assistência em âmbito familiar ${ }^{(28)}$.

Diante do exposto, os estudos analisados permitem inferir que a enfermagem deve prestar uma assistência contínua aos pais de crianças com PC, mediante avaliações periódicas das necessidades centradas na família e, assim, implementar ações para atender a essas necessidades, além de auxiliar na reeducação da sociedade em considerar crianças com disfunções neuromotoras como um membro integrante.

\section{CONCLUSÃO}

A partir dos estudos analisados, evidenciou-se tendência crescente nas publicações um maior destaque para o ano de 2009. Foi observada a prevalência de estudos descritivos e descritivo-exploratório, pesquisas com abordagem qualitativas, com níveis de evidência 4 e 5.

Pode-se constatar que a Enfermagem direciona suas pesquisas nessa temática com maior enfoque ao familiar da criança com PC. Esta revisão constatou que a criança com PC deve estar inserida em um cuidado embasado no conhecimento técnico-científico e na humanização. Recomenda-se um maior investimento em abordagem familiar e social, melhor preparo dos profissionais e reestruturação dos serviços de saúde com estratégias que contribuam para uma melhor interação família-enfermeiro-paciente.

Destaca-se a relevância do tema para a sociedade, visto a PC ser uma doença de alta incidência e com grandes repercussões para a criança e seus familiares. Pretendeu-se fornecer conhecimento acerca desta patologia, tendo como foco os enfermeiros que atuam na área neurológica, possibilitando uma melhor assistência à criança e aos pais, já que estes assumem o papel de cuidadores durante grande parte ou toda a vida da criança.

Procurou-se conceituar a PC, ampliando o conhecimento dos profissionais da saúde, em especial o enfermeiro, visando à melhoria do bem-estar dos pacientes com PC. Nesse sentido, estudos centrados nas intervenções de enfermagem precisam ser realizados para buscar as melhores evidências para uma assistência de qualidade.

\section{REFERÊNCIAS}

1. Dantas MAS, Collet N, Moura FM, Torquato IMB. Impacto do diagnóstico de paralisia cerebral para a família. Texto Contexto Enferm. [Internet] 2010;19(2) [acesso em 10 mai 2012]. Disponível: http://dx.doi. org/10.1590/S0104-07072010000200003

2. Teixeira-Arroyo C, Oliveira SRG. Atividade aquática e a psicomotricidade de crianças com paralisia cerebral. Motriz [Internet] 2007;13(2) [acesso em 12 mai 2012]. Disponível: http://aquabrasil.info/Pdfs/ psicomotricidade.pdf.

3. Lotitto FZ, Rodrigues CC, Ferreira TCD, Caldas MAM. Humanização da assistência de enfermagem para portadores de paralisia cerebral. Saúde Colet. [Internet] 2008;5(23) [acesso em 11 jun 2012]. Disponível: http:// www.redalyc.org/articulo.oa? $\mathrm{id}=84202304$

4. Himpens E, Broeck CV, Oostra A, Calders P, Vanhaesebrouck P. Prevalence, type, distribution and severity of cerebral palsy in relation to gestational age: a meta-analytic review. Dev. Med. Child Neurol. 2008;50 [acesso em 12 jul 2012]. Disponível: http://onlinelibrary. wiley.com/doi/10.1111/j.1469-8749.2008.02047.x/pdf

5. Massi G, Guarinello AC, Santana AP, Paciornik R. Análise clínico-qualitativa do discurso de uma criança com paralisia cerebral. Psicol. estud. [Internet] 2009;14(4) [acesso em 02 ago 2012]. Disponível: http:// dx.doi.org/10.1590/S1413-73722009000400020

6. Ribeiro MFM, Barbosa MA, Porto CC. Paralisia cerebral e síndrome de Down: nível de conhecimento e informação dos pais. Ciênc. saude colet. [Internet] 2011;16(4) [acesso em 13 mai 2012]. Disponível: http:// dx.doi.org/10.1590/S1413-81232011000400009

7. Cerri A, Roehrs H, Crozeta K, Sarquis LMM, Palu L. Problemas éticos no cuidado ao paciente crítico. Cogitare enferm. [Internet] 2011;16(3) [acesso em 03 set 2012]. Disponível: http://ojs.c3sl.ufpr.br/ojs2/index. php/cogitare/article/view/24222/16231

8. Souza MT, Silva MD, Carvalho R. Revisão integrativa: o que é e como fazer. Einstein [Internet] 2010;8(1pt1) [acesso em 20 mai 2012]. Disponível: http://apps. einstein.br/revista/arquivos/PDF/1134-Einsteinv8n1 p102-106_port.pdf

9. Galvão CM. Níveis de Evidência [editorial]. Acta Paul. Enferm. [Internet] 2006;19(2) [acesso em 04 jan 2013]. Disponível: http://www.scielo.br/pdf/ape/v19n2/ a01v19n2.pdf 
10. Gondim KM, Carvalho ZMF. Sentimentos das mães de crianças com paralisia cerebral à luz da Teoria de Mishel. Esc. Anna Nery [Internet] 2012;16(1) [acesso em 14 set 2012]. Disponível: http://dx.doi.org/10.1590/ S1414-81452012000100002

11. Jan MMS. Cerebral Palsy: comprehensive review and update. Ann Saudi Med. [Internet] 2006;26(2) [acesso em 10 mai 2012]. Disponível: http://www.kau.edu.sa/ Files/140/Researches/11821_cerebral_palsy.pdf

12. Souza KES, Sankako AN, Carvalho SMR, Braccialli LMP. Classificação do grau de comprometimento motor e do índice de massa corpórea em crianças com paralisia cerebral. J. Hum. Growth Dev. [Internet] 2011;21(1) [acesso em 16 jun 2012]. Disponível: http://pepsic. bvsalud.org/pdf/rbcdh/v21n1/02.pdf

13. Silveira RD, Motta MCS. Assistência à criança com disfunção neuromotora na abordagem da rede social: um estudo de caso. Esc. Anna Nery [Internet] 2009;13(1) [acesso em 03 jun 2012]. Disponível: http://dx.doi. org/10.1590/S1414-81452009000100013

14. Chagas PSC, Deflipo EC, Lemos RA, Mancini MC, Frônio JS, Carvalho RM. Classificação da função motora e do desempenho funcional de crianças com paralisia cerebral. rev. bras. fisioter. [Internet] 2008;12(5) [acesso em 03 jun 2012]. Disponível: http:// dx.doi.org/10.1590/S1413-35552008000500011

15. Haak P, Lenski M, Hidecker MJC, Li M, Paneth N. Cerebral palsy and aging. Dev. Med. Child Neurol. [Internet] 2009;51(Suppl 4) [acesso em 01 jun 2012]. Disponível: http://onlinelibrary.wiley.com/doi/10.1111/ j.1469-8749.2009.03428.x/pdf

16. Buran CF, Sawin K, Grayson P, Criss S. Family needs assessment in cerebral palsy clinic. J Spec Pediatr Nurs. [Internet] 2009;14(2) [acesso em 15 set 2012]. Disponível: http://onlinelibrary.wiley.com/doi/10.1111/ j.1744-6155.2008.00176.x/pdf

17. Sigurdardottir S, Vik T. Speech, expressive language and verbal cognition of preschool children with cerebral palsy in Iceland. Dev. Med. Child Neurol. [Internet] 2011;53 [acesso em 08 set 2012]. Disponível: http://onlinelibrary. wiley.com/doi/10.1111/j.1469-8749.2010.03790.x/pdf

18. Prudente COL, Barbosa MA, Porto CC. Relação entre a qualidade de vida de mães de crianças com paralisia cerebral e a função motora dos filhos, após dez meses de reabilitação. Rev. Latino-Am. Enfermagem [Internet] 2010;18(2) [acesso em 08 jul 2012]. Disponível: http:// dx.doi.org/10.1590/S0104-11692010000200002
19. Brasileiro IC, Moreira TMM, Jorge MSB, Queiroz MVO, Mont'Alverne DGB. Atividades e participação de crianças com paralisia cerebral conforme a Classificação Internacional de Funcionalidade, Incapacidade e Saúde. Rev. bras. enferm. [Internet] 2009;64(4) [acesso em 10 jun 2012]. Disponível: http://dx.doi.org/10.1590/S003471672009000400002

20. Parkes J, Mccollough N, Madden A, Mccahey E. The health of children with cerebral palsy and stress in their parents. J Adv Nurs. [Internet] 2009;65(11) [acesso em 01 set 2012]. Disponível: http://www.ncbi.nlm.nih.gov/ pubmed/19737327

21. Oliveira ANA, Golin MO, Cunha MCB. Aplicabilidade do Sistema de Classificação da Função Motora Grossa (GMFCS) na paralisia cerebral: revisão de literatura. Arq Bras Cienc Saúde. [Internet] 2010;35(3) [acesso em 29 mai 2012]. Disponível: http://files.bvs.br/ upload/S/1983-2451/2010/v35n3/a1690

22. Pfeifer LI, Silva DBRS, Funayama CAR, Santos JL. Classification of cerebral palsy: association between gender, age, motor type, topography and Gross Motor Function. Arq Neuropsiquiatr. [Internet] 2009;67(4) [acesso em 01 jun 2012]. Disponível: http://dx.doi. org/10.1590/S0004-282X2009000600018

23. Reid SM, Carlin JB, Reddihough DS. Distribution of motor types in cerebral palsy: how do registry data compare? Dev Med Child Neurol. [Internet] 2011;53 [acesso em 02 jul 2012]. Disponível: http:/onlinelibrary. wiley.com/doi/10.1111/j.1469-8749.2010.03844.x/pdf

24. Singh BK, Masey H, Morton R. Levels of continence in children with cerebral palsy. Pediatr Nurs. [Internet] 2006;18(4) [acesso em 12 jul 20120]. Disponível: http://web.ebscohost.com/ehost/pdfviewer/ pdfviewer?sid=7a098987-1043-48f5-aafa-a4869786eef f\% $\%$ sessionmgr10\&vid=2\&hid $=14$

25. Inácio IF, Cardoso RCS, Nunes LGP. A relevância da ação da enfermagem na inclusão social dos portadores de paralisia cerebral. Rev. Edu., Meio Amb e Saúde. [Internet] 2008;3(1) [acesso em 02 ago 2012]. Disponível: http://www.faculdadedofuturo.edu.br/ revista/2008/pdfs/REMAS3(1)177a188.pdf

26. Milbrath VM, Soares DC, Amestoy SC, Cecagno $\mathrm{D}$, Siqueira $\mathrm{HCH}$. Mães vivenciando o diagnóstico de paralisia cerebral em seus filhos. Rev. Gaúcha Enferm. [Internet] 2009;30(3) [acesso em 12 jul 2012]. Disponível: http://seer.ufrgs.br/index. php/RevistaGauchadeEnfermagem/article/ view/7733/6965 
27. ParkesJ,HillN. Theneeds of children andyoung peoplewith cerebral palsy. Pediatr Nurs. [Internet] 2010;4(22) [acesso em 08 set 2012]. Disponível: http://web.ebscohost.com/ ehost/pdfviewer/pdfviewer?sid=251d5c4b-df5f-4bebad75-c5c7940f3e0f\%40sessionmgr14\&vid=2\&hid=14

28. Vasconcelos VM, Frota MA, Pinheiro AKB, Gonçalves MLC. Percepção de mães acerca da qualidade de vida de crianças com paralisia cerebral. Cogitare enferm. [Internet] 2010;15(2) [acesso 12 out 2012]. Disponível: http://ojs.c3sl.ufpr.br/ojs2/index.php/cogitare/article/ view/17853/11648 